\title{
Surgical or nonsurgical treatment for teeth with existing root filings?
}

\author{
Abstracted from \\ Torabinejad M, Corr R, Handysides R, Shabahang S. \\ Outcomes of nonsurgical retreatment and endodontic surgery: a systematic review. \\ J Endod 2009; 35: 930-937 \\ Address for correspondence: Mahmoud Torabinejad, Endodontic Residency Program, \\ Department of Endodontics, School of Dentistry, Loma Linda University, Loma Linda CA 92350, USA. \\ E-mail: mtorabinejad@llu.edu
}

\section{Questions: In symptomatic patients who have previously had endodontic treatment, is nonsurgical treatment more effective than endodontic surgery?}

Data sources Data was sought using Medline and the Cochrane Library, and relevant chapters from three major endodontic texts were consulted: Principles and Practice of Endodontics (4th Edn; editors; Torabinejad and Walton; 2008); Pathways of the Pulp (9th edition; editors, Cohen and Hargreaves; 2006), and Endodontics (6th edition; editors, Ingle, Bakland and Baumgartner; 2008). Every issue was also searched of the most recent 2 years of the following major endodontic journals: International Endodontic Journal; Journal of Endodontics; and Oral Surgery, Oral Medicine, Oral Pathology, Oral Radiology and Endodontology along with the reference lists of identified articles.

Study selection Articles were included from peer-reviewed journals if they were published in English, and reported clinical and/ or radiographic outcome data for nonsurgical endodontic retreatment or for endodontic surgery, with followup data for a minimum of 25 teeth and a minimum 2-year mean followup period. Studies reporting outcomes based on individual roots as opposed to whole teeth, or that did not report clinical or radiographic outcomes, which were animal studies, or studies that reported histological data only, were excluded.

Data extraction and synthesis When necessary, the reviewers recalculated success and failure rates when they were not directly provided in papers' tables or in the text, or when only particular data subsets met the inclusion criteria. To facilitate meta-analysis, the data were standardised according to a commonly applied classification system used to assess outcomes for nonsurgical retreatment and surgical endodontics: (1) Complete healing; (2) Incomplete healing; (3) Uncertain healing; and (4) Unsatisfactory healing (failures).

When uncertainty existed regarding which of the above four categories correlated with those reported in a given article, the data were assigned to the lower healing category. For this review, success was defined as teeth categorised as showing complete healing or incomplete healing. Weighted success rates, pooled success rates, and 95\% confidence interval $(\mathrm{Cl})$ estimates of outcomes were generated in the metaanalysis from compiled data from the included studies by using the DerSimonian-Laird random effects pooling method.

Results Twenty-six endodontic surgery and eight nonsurgical retreatment articles were included. There were only four randomised controlled trials (one in the nonsurgical retreatment, and three in the endodontic surgery group). A significantly higher success rate was found for endodontic surgery at 2-4 years (77.8\%) than for nonsurgical retreatment for the same followup period (70.9\%; $\mathrm{P}<0.05)$. At 4-6 years, however, this relationship was reversed, with nonsurgical retreatment showing a higher success rate of $83.0 \%$, compared with $71.8 \%$ for endodontic surgery $(P<0.05)$. Insufficient numbers of articles were available to make comparisons after 6 years of followup. Endodontic surgery studies showed a statistically significant decrease in success with each increasing followup interval $(P<0.05)$. The weighted success for 2-4 years was $77.8 \%$, which declined at $4-6$ years to $71.8 \%$ and further declined at $>6$ years to $62.9 \%(P<0.05)$. Conversely, the nonsurgical retreatment success rates demonstrated a statistically significant increase in weighted success at $2-4$ years $(70.9 \%)$ versus 4-6 years (83.0\%; $\mathrm{P}<0.05)$.

Conclusions On the basis of these results it appears that endodontic surgery offers more favourable initial success, but nonsurgical retreatment offers a more favourable long-term outcome.

\section{Commentary}

Despite the recent advances in endodontic therapy, nearly $10 \%$ of failures still occur after initial root canal treatment. ${ }^{1}$ Thus, the evidence-based decisionmaking for previously treated teeth is an important issue for endodontic treatment. Since only limited numbers of controlled trials have been reported, it is difficult to make a clear argument supporting either nonsurgical retreatment or endodontic surgery as the treatment of choice for secondary root canal treatment.

This article is a unique review in comparing the clinical significance of nonsurgical retreatment and endodontic surgery, not by direct comparison, but partially by the extrapolation of observational studies of one arm of treatment modality. Cautious extrapolation from these results is required because of the difference in participants and techniques employed in these studies.

The conclusion derived from this review, that endodontic surgery offers more favourable initial success but nonsurgical retreatment offers a more favourable long-term outcome, was quite reasonable. This phenomenon may be explained by slower healing dynamics of peri-apical tissue after the nonsurgical approach and the late failures in part caused by retrograde fillings following the surgical approach. ${ }^{2}$

A conventional endodontic retreatment approach is judged to be the most appropriate in the first instance, providing that access 
to the root canal is possible. This does not preclude a subsequent surgical approach. A surgical approach to a poorly filled root would require orthograde filling prior to surgery, anyhow.

Evidence-based medicine is the integration of clinical expertise, patient values and the best evidence in the decisionmaking process for patient care. ${ }^{3}$ Endodontic treatment is the accumulation of clinical expertise. A clinician skilled in root canal therapy is not always expert at peri-apical surgery. Cost and time can form a patient's view of the value and outcome, although long-term survival is the most important outcome in endodontic therapy. With endodontic surgery, the treated root is definitely shortened by surgical procedures, and repeated surgery is not usually recommended. Why do we have to choose endodontic surgery in the first instance when a conventional endodontic retreatment approach is available? Parallel comparison of endodontic surgery and retreatment has little meaning clinically.

\section{Toru Naito}

Department of Geriatric Dentistry , Fukuoka Dental College, Tamura 2-15-1, Sawara, Fukuoka, Japan

1. Imura N, Pinheiro ET, Gomes BP, Zaia AA, Ferraz CC, Souza-Filho FJ. The outcome of endodontic treatment: a retrospective study of 2000 cases performed by a specialist. J Endod 2007; 33: 1278-1282.

2. Jesslén $P$, Zetterqvist $L$, Heimdahl A. Long-term results of amalgam versus glass ionomer cement as apical sealant after apicectomy. Oral Surg Oral Med Oral Pathol Oral Radiol Endod 1995; 79: 101-103.

3. Sackett D. Evidence-based Medicine: How to Practice and Teach EBM. 2nd Edn. Edinburgh: Churchill Livingstone; 2000.

Evidence-Based Dentistry (2010) 11, 54-55. doi:10.1038/sj.ebd.6400724 